\title{
Performance Comparison of Organic Rankine Cycle and Vapour Compression Cycle Hybrid Cooling-Heating System using Subcritical or Supercritical Working Fluid
}

\author{
Yam Ke San, Raja Wahiduzzaman Bin Raja Ismail , Vincent Lee Chieng Chen , and Jung Hyung Chul \\ Department of Mechanical Engineering, Curtin University Malaysia, CDT 250 Miri Sarawak Malaysia.
}

\begin{abstract}
This paper presents a mathematical modelling on the evaluation of cooling, heating and power performance of a hybrid system of Organic Rankine Cycle and Vapour Compression Cycle. The system is assumed to be powered through solar parabolic trough collector and is able to generate a cooling power of $10 \mathrm{~kW}$. Refrigerants R134a or R245fa are chosen as the working fluid of the system. The system is constructed using commercial energy modelling tool AspenPlus. Analysis is performed to determine the effect of changing the mass flow rate split ratio on the energy output. The effect of using subcritical and supercritical working fluid is also compared. Particular attention is paid toward the condition where the power output is equivalent to the energy consumption in view of creating a selfpowered cooling and heating system. The result shows that the coefficient of performance for system using R245fa is higher compared to that using R134a. However, the systen using R134a allows a self-powered cooling and heating system to be achieved to be achieve at a much higher mass split ratio, resulting the system to be $35 \%$ more efficient in the

performance.
\end{abstract}

\section{Introduction}

Air conditioning contributes to approximately $15 \%$ of the total energy consumption in Malaysia [1], that mainly comes from coal-fueled power plants with negative carbon footprints. As a measure to curb the consumption of non-renewable and carbon emission, this study looks at the feasibility of the Organic Rankine Cycle-Vapor Compression Cycle (ORC-VCC) system as a green energy powered hybrid system that provide air cooling and water heating for household application.

The use of Organic Rankine Cycle to harvest power from low-grade heat source is not new and has been known to be a viable technology for converting heat to electrical power in various of the system that ranges from micro-scale vehicle exhaust system [2] to large-scale $30 \mathrm{MW}$ of geothermal power plant [3]. Increasing interest in renewable energy worldwide has prompted significant research and development in the field. One drawback of the system that researchers have been addressing is its low efficiency. Research have shown that the efficiency can be significantly improved by altering or revamping the cycle architectures $[4,5]$, or by optimizing the selection of working fluid [6,7]. Another increasingly popular approach to improve the efficiency is through cogenerations. Cogeneration minimizes the system size by combining the ORC with other energy system and converts all form of energy exchanges within the cycles into useful output. Some examples of cogeneration unit include combining the waste heat from biomass combustion and the solar power to increase the critical temperature [8], integrating the solar-powered ORC to a desalination process $[9,10]$, integrating ORC into solid fuel cell and micro-gas turbine gasification [11], and combining ORC to a Vapor Compression Cycle (VCC) to produce both cooling and heating [12].

The research on ORC-VCC is still relatively new. A recent study [12] evaluated the feasibility of a combined biomass and solar powered ORC-VCC in providing cooling and water heating in Greece during the summer season and concluded the feasibility of the system. However, the study uses the subcritical working fluid and did not report the effect of the mass flow rate ratio. Understanding the effect of the mass flow rate ratio on the energy balance is critical for designing an optimum system. In addition, the selection of the working fluid condition also is known to be pivotal in the system efficiency [4]. This study evaluates the effect of the mass flow rate ratio and the performance of the subcritical and supercritical working fluid $\mathrm{R} 245 \mathrm{fa}$ in a solar-powered ORC-VCC.

\section{Methodology}

\subsection{System description}

We consider a ORC-VCC system consists of a solarpowered ORC that is interconnected to the VCC system. The ORC system consists of a solar heater, an expander, 
a pump and a condenser, whereas the VCC system consist of an evaporator, a compressor, a condenser, and an expansion valve, as shown in Fig. 1. Both systems share the same condenser, which means the flows from both systems joins together to enter the condenser, and then are split upon exiting the condenser. The primary function of the ORC system is to convert the solar heat into power, that is used to drive the motor of the compressor in the VCC system. In this case compressor is the only component that consumes significant power. Pump typically requires very little work to operate and its energy consumption can be neglected. The VCC system functions as an air cooler, which absorbs the heat through the evaporator. The thermodynamic system is closed by having the condenser as a heat remover. The heat taken out from the condenser is utilized for heating water. Therefore, the whole system operates as a cogeneration unit (cooling and heating). The detail of the flow sequences is listed in Table 1 .

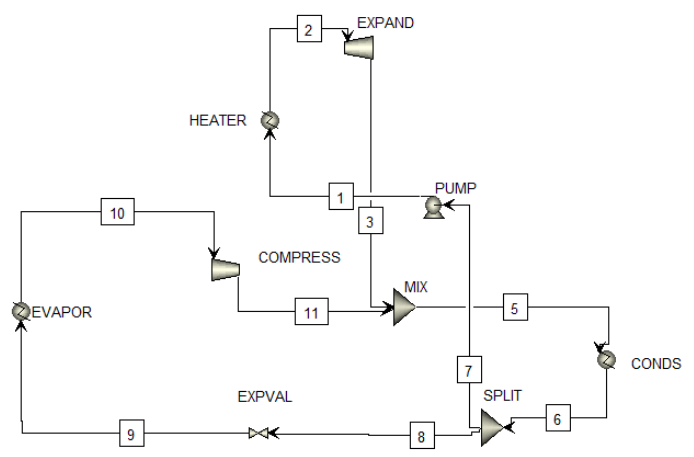

Figure 1. Components of the ORC-VCC system developed in Aspen Plus.

Table 1. The state point of the ORC-VCC.

\begin{tabular}{|c|c|}
\hline State point & Description \\
\hline $1-2$ & Inlet and outlet of the solar PTC \\
\hline $2-3$ & Inlet and outlet of the turbine expander \\
\hline$\{3,11\}-5$ & Inlet ad outlet of the mixture \\
\hline $5-6$ & Inlet and outlet of the condenser \\
\hline $6-\{7,8\}$ & Inlet and outlet of the splitter \\
\hline $7-1$ & Inlet and outlet of the pump \\
\hline $8-9$ & Inlet and outlet of the expansion valve \\
\hline $9-10$ & Inlet and outlet of the evaporator \\
\hline $10-11$ & Inlet and outlet of the compressor \\
\hline
\end{tabular}

\subsection{System constraints}

The key operational parameters are the temperature and the pressure of the working fluid at the inlet of the ORC expander, and the condensation temperature/pressure. For direct vapour generation saturation operation, the maximum operating temperature of a Parabolic Trough Collector (PTC) is $250{ }^{\circ} \mathrm{C}$ [9]. The pressure at the heater is set at $3 \mathrm{MPa}$ for subcritical and $6 \mathrm{MPa}$ for supercritical fluid. The condensation temperature for the system is set at $80^{\circ} \mathrm{C}$, in order to cover domestic hot water demands. The evaporation temperature of the VCC is set equal to $10^{\circ} \mathrm{C}$, which is reasonably low for air cooling purpose. The total mass flow rate of the system $\dot{m}_{t}$ is set at $0.32 \mathrm{~kg} / \mathrm{s}$. Finally, the cooling load has to be specified but the value depends on the applications. Many factors such as the weather, and the building characteristics (size, material etc) can influence the cooling demand of a building. A heating load of $10 \mathrm{~kW}$ is specified to the evaporator. The average Malaysia electricity consumption is about $1 \mathrm{~kW}$ [13]. Therefore, the system can provide cooling up to ten households. In order for the system to be able to generate sufficient cooling power without relying on the grid electricity, the ORC must generate sufficient electricity power for the operation of the compressor and the pump. This requires a balance of the mass flow rate in the ORC and the VCC cycle. Therefore, this work aims to analyze the effect of changing the balance of the mass flow rate in the system, i.e. the ratio of the mass flow rate into the VCC $\dot{m}_{c}$ to the total mass flow rate $\dot{m}_{t}$,

$$
R_{m}=\frac{\dot{m}_{c}}{\dot{m}_{t}}
$$

, and also to compare the performance of the cycle using subcritical and supercritical fluid. Equation (1) is termed as mass split ratio.

The selection of suitable working fluid is essential to ensure high thermal efficiency and high utilization of the available heat source. Two commonly employed refrigerants, i.e. R134a and R245fa are chosen as the working fluid for the organic Rankine cycles given its desirable thermodynamic and thermal transport properties such as low boiling temperature and relatively high thermal conductivity, which makes it an ideal for low to medium temperature applications. The dry nature of the fluid ensure that the fluid remains in superheated state at the end of the expansion. In addition, it is nonflammable, has very low toxicity, non-corrosive and is environmentally friendly. The properties of both fluids are listed in Table 2.

Table 2 Physical, safety and environmental data of the working fluids used this [6]

\begin{tabular}{|c|c|c|}
\hline Properties & R134a & R245fa \\
\hline Molecular mass (kg/kmol) & 102.03 & 134.05 \\
\hline Critical Temperature (K) & 374 & 427.2 \\
\hline Critical Pressure (K) & 4.059 & 3.64 \\
\hline Atmospheric lifetime & 14.0 & 7.2 \\
\hline Global Warming Potential & 1430 & 900 \\
\hline Ozone depletion potential & 0 & 0 \\
\hline
\end{tabular}




\subsection{Mathematical modelling}

The performance of the system is evaluated using first and second law of thermodynamics. The system is assumed to operate under steady-state assumption. For simplicity, pressure drops and heat losses in the equipment and the pipes are neglected. The total heating generated from a solar collector can be expressed as

$$
Q_{s}=\dot{m}_{p}\left(h_{1}-h_{2}\right)
$$

where $\dot{m}_{p}$ represents mass flow rate of working fluid in the ORC cycle, and the power generation at turbine expander is

$$
W_{t}=\dot{m}_{p}\left(h_{2}-h_{3}\right) \eta_{t} \eta_{m}
$$

where $\eta_{t}$ is the isentropic efficiency and $\eta_{m}$ is the mechanical efficiency for the expander. The energy consumption of the pump is

$$
W_{p}=\dot{m}_{p}\left(h_{7}-h_{8}\right) \eta_{p}
$$

The amount of heat energy absorbed by the evaporator of the VCC is

$$
Q_{e}=\dot{m}_{c}\left(h_{10}-h_{11}\right)
$$

The energy consumption of the compressor is

$$
W_{c}=\dot{m}_{c}\left(h_{11}-h_{12}\right) \eta_{c}
$$

The total energy consumption of the system therefore is $W_{T}=W_{c}+W_{p}$. The heat removal by the condenser is

$$
Q_{c}=\dot{m}_{t}\left(h_{5}-h_{6}\right)
$$

where $\dot{m}_{t}=\dot{m}_{p}+\dot{m}_{c}$. The coefficient of performance (COP) for the VCC is

$$
C O P_{V C C}=\frac{Q_{c}}{W_{C}}
$$

The net COP for the system is

$$
C O P_{N e t}=\frac{Q_{e}+Q_{c}}{Q_{s}}
$$

The efficiency of the ORC is

$$
\eta_{p}=\frac{W_{t}}{Q_{s}}
$$

The ratio of the turbine power output to the energy consumption is

$$
R_{w}=\frac{w_{t}}{W_{c}+W_{p}}
$$

The ratio of the cooling and heating energy is

$$
R_{h}=\frac{Q_{e}}{Q_{c}}
$$

The value for the efficiency of the system components is listed in Table 3. The system is constructed in a commercial energy simulator AspenPlus, in which the thermodynamic calculation is performed.

Table 3 Efficiency of the system component [14].

\begin{tabular}{|c|c|}
\hline Assumption & Value \\
\hline Pump isentropic efficiency $\eta_{p}(\%)$ & 65 \\
\hline Expander isentropic efficiency $\eta_{t}(\%)$ & 60 \\
\hline Compressor isentropic efficiency $\eta_{c}(\%)$ & 75 \\
\hline Mechanical efficiency $\eta_{m}(\%)$ & 98 \\
\hline
\end{tabular}

\section{Result \& discussion}

Figure 2 shows the P-h diagram for the ORC-VCC cycle. Using supercritical fluid increases the area of the ORC system, which increases the expander output. By doubling the pressure in the boiler, the expander output is tripled. The effect of using supercritical on the temperature-entropy diagram is also shown in Figure 2. The area of the ORC is doubled, which allows the fluid to reach the maximum temperature $\left(250{ }^{\circ} \mathrm{C}\right)$ at a lower value of entropy and increases the expander power output.
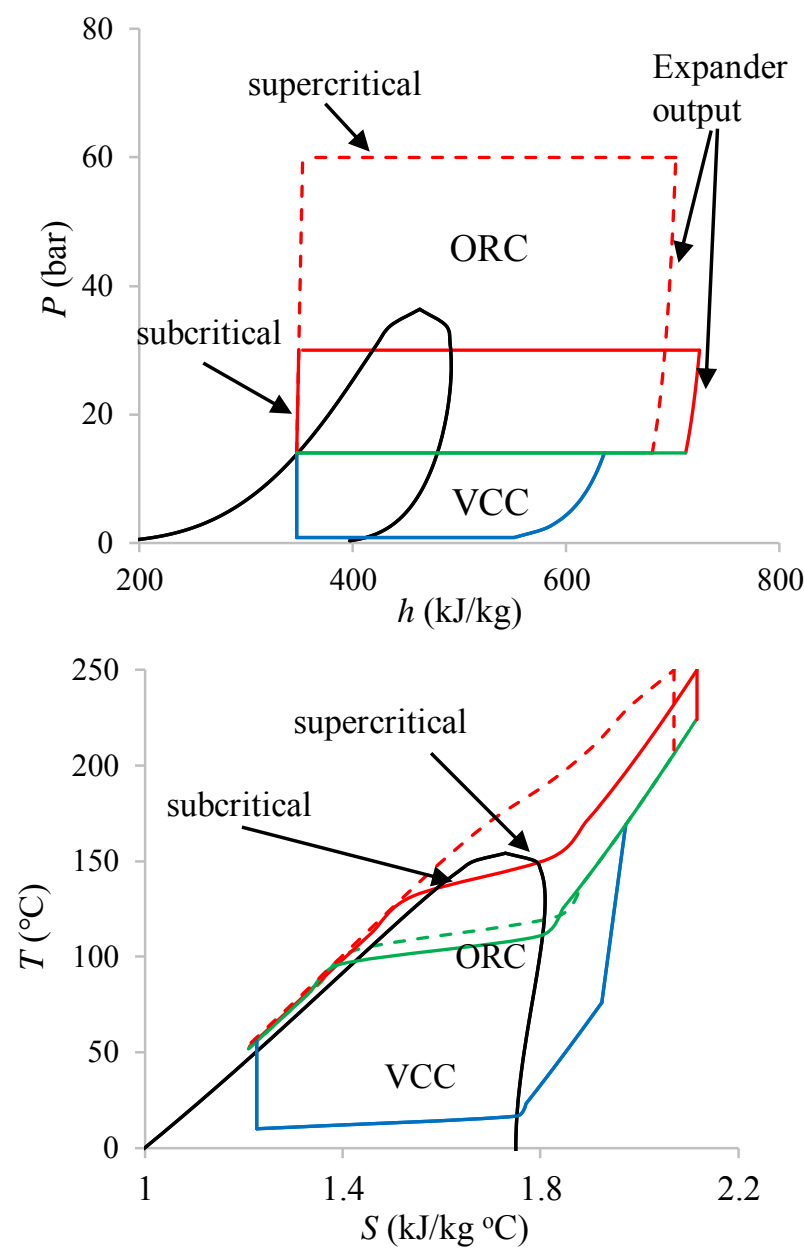

Figure 2 (top) Pressure-enthalpy, (bottom) temperature-entropy diagram for the ORC-VCC system using R245fa. 
Figure 3 shows the effect of the mass flow rate split ratio on the total work consumption, and the expander power output for both subcritical and supercritical cycle. Increasing the flow rate into the VCC system linearly increases the consumption, whereas the lower flow rate into the ORC cycle linearly decreases the power output. It can be seen that employing supercritical cycle doubled the turbine output at $R_{m}=0.1$. However, the rate of decrease of the turbine energy also increases for supercritical cycle, which decreases its difference from the subcritical case at large $R_{m}$. On other hand, there is no significant changes to the consumption energy for both cycles.

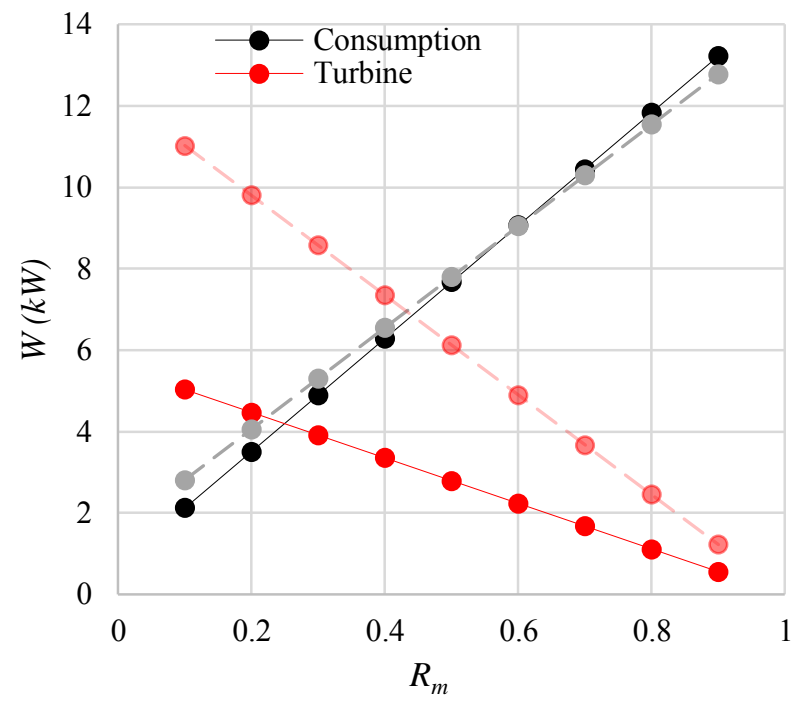

Figure 3. $W_{T}$ and $W_{t}$ as function of $R_{m}$ for subcritical (bold lines) and supercritical cycle (dashed lines) using R134a.

In order to design a self-powered $10 \mathrm{~kW}$ cooling system, we seek for the flow rate split ratio $R_{m}$ that could yield a cooling load of $10 \mathrm{~kW}$ at the ratio of the turbine power output to the energy consumption $R_{w} \geq 1$. When $R_{w}=1$, the system produces a power output equal to its consumption, and an ideal maximum cooling power. When $R_{w} \geq 1$, the system produces cooling less than its ideal maximum, but also a surplus of electricity. When $R_{w}<1$, the system produces a cooling load greater than its ideal maximum but requires additional power from external source in order to operate. Clearly it is desirable

to achieve the work ratio $R_{w}=1$ at a large value of the flow rate split ratio $R_{m}$ since it improves the system efficiency and provides a greater turbine output. This can be seen in Fig. 4 which illustrates the relationship between $R_{w}$ and $R_{m}$ for both refrigerants using subcritical or supercritical cycles. In all cases, $R_{w}$ decreases exponentially against $R_{m}$, for both subcritical and supercritical fluid. Also all have very similar gradients, in the range of $\mathrm{e}^{-4.3 \mathrm{Rm}}$ to $\mathrm{e}^{-4.8 \mathrm{Rm}}$. Since the gradient of both lines are approximately the same, the difference between subcritical and supercritical can be inferred by subtracting the equation of both lines. Therefore, the supercritical cycle is consistently offset the subcritical by a constant value, giving supercritical cycle having a greater value of $R_{m}$ at $R_{w}=1$. For system using R245fa, supercritical cycle increases the value of
$R_{m}$ from 0.1 to 0.2 . For system using R124a, supercritical cycle greatly increases the value of $R_{m}$ from 0.28 to 0.42 .
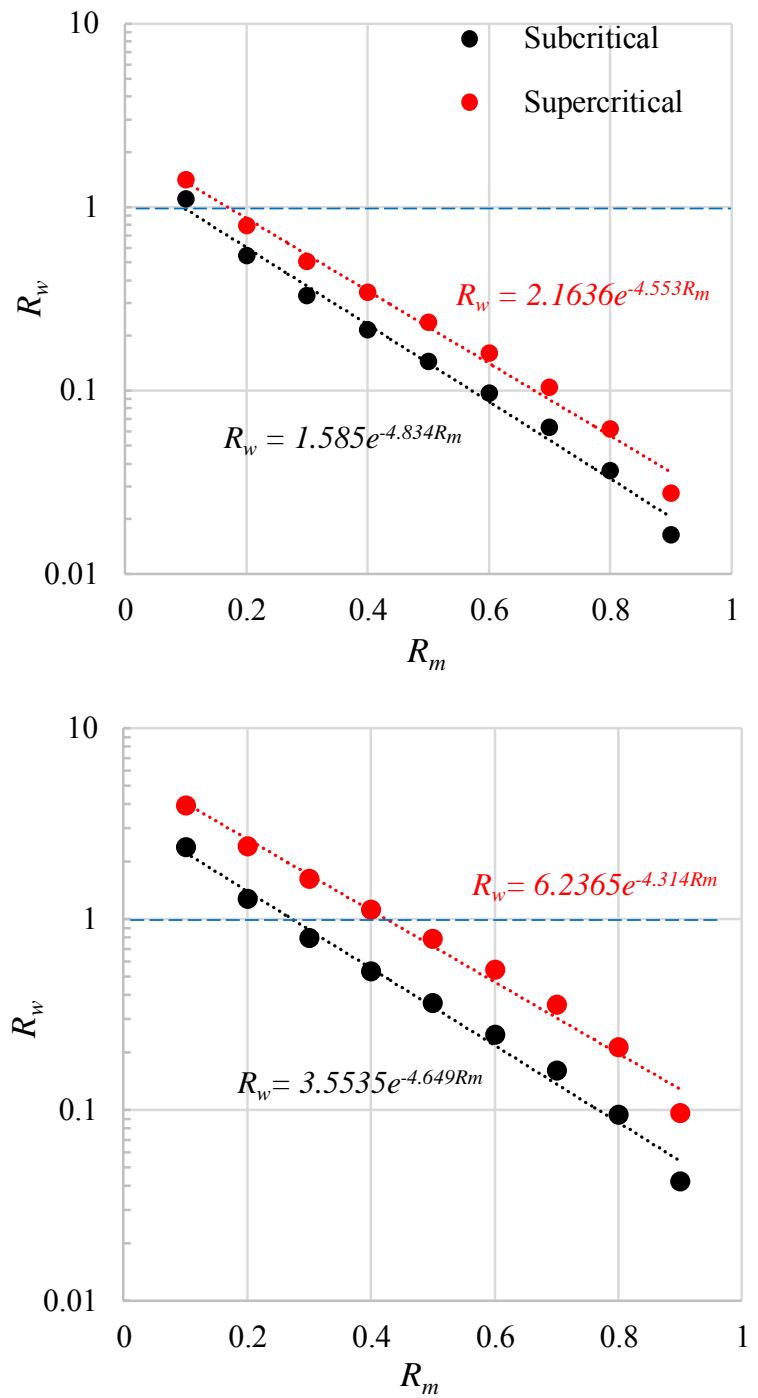

Figure 4. $R_{w}$ as function of $R_{m}$ for system using R245fa (top) and R134a (bottom). The blue dashed line denotes $R_{w}=1$.

The efficiency of the system is evaluated using the coefficient of performance (COP). The gross COP computes the efficiency in the conversion of the heat source to the total useful output (both cooling and heating $\left.Q_{c}+Q_{e}\right)$. As shown in Figure 5, the COP increases exponentially with the increase of $R_{m}$ for both refrigerants in either subcritical or supercritical cycle, i.e. the system is highly efficient at very large $R_{m}$. The use of supercritical increases the COP slightly. Also, in overall, R245fa produces slightly a higher COP than R134a. However, for the case $R_{w}=1$, the low value of $R_{m}$ for R245fa results in the low COP. The COP of supercritical and subcritical for R245fa at $R_{w}=1$ is 1.3 and 1.1, respectively. Therefore, the supercritical cycle increases the COP by 0.2 , which is about $18 \%$ higher than subcritical cycle. On the other hand,

the high value of $R_{m}$ for R134a results in a higher COP. The COP of supercritical and subcritical for R134a at $R_{w}=1$ is 1.75 and 1.35 , respectively. Therefore, the supercritical cycle increases the COP by 0.4 , an 
increment of $30 \%$. It can be concluded that R134a is more efficient given that the large value of $R_{m}$ at $R_{w}=1$, and possessing a greater value of COP, $35 \%$ greater than that of R245fa.

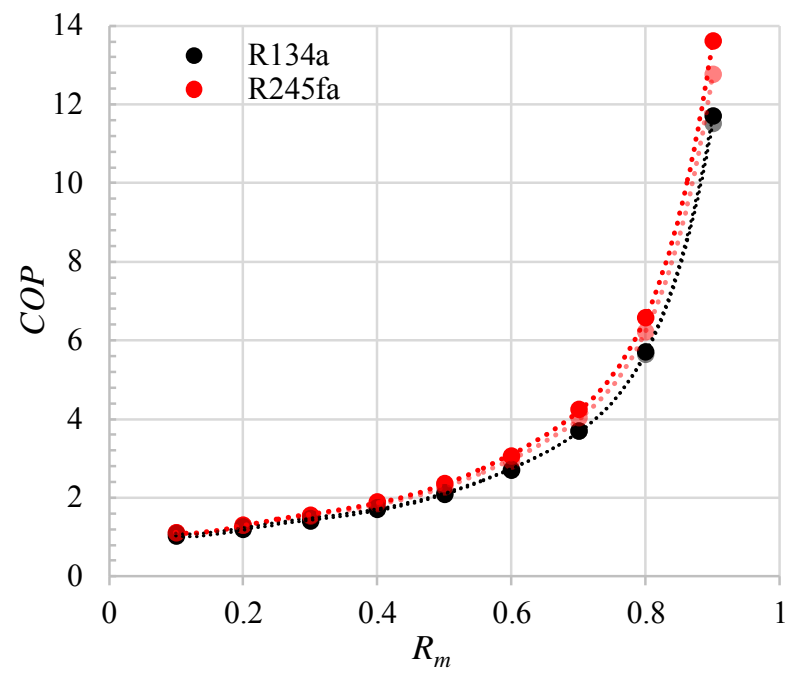

Figure 5. COP as function of ratio $R_{m}$ for R134a and R245f in subcritical (faded colour) or supercritical (bold colour) mode.

\section{Conclusion}

This work presents a mathematical evaluation on the cooling, heating and power performance of a combined system of Organic Rankine Cycle and Vapour Compression Cycle. The work focusses on the effect of changing the mass flow rate split ratio $R_{m}$ on the energy output and compare the effect of using subcritical and supercritical working fluid. Particular attention is paid toward the scenario where the ratio of the turbine power output to the energy consumption $R_{w}$ is equal to one, in view of creating a self-powered cooling and heating system. The following conclusions are drawn:

1. $R_{w}$ decreases exponentially against an increasing $R_{m}$ at a fixed gradient of approximately $\mathrm{e}^{-4.6 R m}$ for both R134a and R245fa. Supercritical cycle is found to be consistently offset the subcritical cycle by a constant magnitude, allowing $R_{w}=1$ to be achieved at higher $R_{m}$.

2. The net coefficient of performance COP increases exponentially with the increase of $R_{m}$ for both R134a and R245fa. The COP is higher with the use of $\mathrm{R} 245 \mathrm{fa}$ compared to the COP of R134a. The COP is also slightly increased when using supercritical cycle.

3. R134a is recommended as the working fluid for the system, given its larger value of $R_{m}$ at $R_{w}=1$. In supercritical mode, the COP of R134a is $35 \%$ higher than that of R145fa.

\section{References}

1. T. Kubota, S. Jeong, D.H.C. Toe, D.R. Ossen. Energy consumption and air-conditioning usage in residential buildings of Malaysia. Journal of international Development and Cooperation, 17(3), pp.61-69. (2004)

2. T. Wang, Y. Zhang, Z. Peng, G. Shu. A review of researches on thermal exhaust heat recovery with Rankine cycle. Renewable and sustainable energy reviews, 15(6), pp.2862-2871. (2011)

3. M. Yari. Exergetic analysis of various types of geothermal power plants. Renewable Energy, 35(1), 112-121. (2010)

4. S. Lecompte, H. Huisseune, M. Van Den Broek, B. Vanslambrouck, M. De Paepe, M. Review of organic Rankine cycle (ORC) architectures for waste heat recovery. Renewable and Sustainable Energy Reviews, 47, pp.448-461. (2015)

5. S. Quoilin, M. Orosz, H. Hemond, \& V. Lemort. Performance and design optimization of a low-cost solar organic Rankine cycle for remote power generation. Solar energy, 85(5), 955-966. (2011)

6. B.F. Tchanche, G. Papadakis, G. Lambrinos and A. Frangoudakis. Fluid selection for a low-temperature solar organic Rankine cycle. Applied Thermal Engineering, 29(11-12), pp.2468-2476. (2009)

7. Y. Dai, J. Wang and L. Gao. Parametric optimization and comparative study of organic Rankine cycle (ORC) for low grade waste heat recovery. Energy Conversion and Management, 50(3), pp.576-582. (2009)

8. J. Wang, J., Y. Yang. Energy, exergy and environmental analysis of a hybrid combined cooling heating and power system utilizing biomass and solar energy. Energy Conversion and Management, 124, pp.566-577. (2016)

9. A.S. Nafey, M.A. Sharaf. Combined solar organic Rankine cycle with reverse osmosis desalination process: Energy, exergy, and cost evaluations. Renewable Energy, 35(11), pp.2571-2580. (2010)

10. C. Li, G. Kosmadakis, D. Manolakos, E. Stefanakos, G. Papadakis, \& D.Y. Goswami. Performance investigation of concentrating solar collectors coupled with a transcritical organic Rankine cycle for power and seawater desalination cogeneration. Desalination, 318, 107-117. (2013).

11. M. Ebrahimi and I. Moradpoor. Combined solid oxide fuel cell, micro-gas turbine and organic Rankine cycle for power generation (SOFC-MGTORC). Energy Conversion and Management, 116, pp.120-133. (2016)

12. S. Karellas, K. Braimakis. Energy-exergy analysis and economic investigation of a cogeneration and trigeneration ORC-VCC hybrid system utilizing biomass fuel and solar power. Energy conversion and management, 107, pp.103-113. (2016)

13. T. Kubota, S. Jeong, D.H.C. Toe, \& D.R. Ossen. Energy consumption and air-conditioning usage in residential buildings of Malaysia. Journal of international Development and Cooperation, 17(3), 61-69. (2011)

14. K. Braimakis, A. Thimo and S. Karellas. Technoeconomic analysis and comparison of a solarbased biomass ORC-VCC system and a PV heat pump for domestic trigeneration. Journal of Energy Engineering, 143(2), p.04016048. (2016) 